\title{
Editorial: Virtual Reality and Mental Health: Opportunities to Advance Research and Practice
}

\author{
Nilufar Baghaei ${ }^{1,2 *}$, Hai-Ning Liang ${ }^{3}$, Mark Billinghurst ${ }^{4,5}$, John Naslund $^{6}$ and \\ Oyewole Oyekoya ${ }^{7}$
}

${ }^{1}$ Massey University, Auckland, New Zealand, ${ }^{2}$ The University of Queensland, Brisbane, QLD, Australia, ${ }^{3} X i$ 'an Jiaotong-Liverpool University, Suzhou, China, ${ }^{4}$ University of South Australia, Adelaide, SA, Australia, ${ }^{5}$ Auckland Bioengineering Institute, The University of Auckland, Auckland, New Zealand, ${ }^{6}$ Harvard Medical School, Boston, MA, United States, ${ }^{7}$ Hunter College (CUNY), New York, NY, United States

Keywords: mental health, virtual reality, virtual reality therapy, extended reality, design, evaluation

Editorial on the Research Topic

Virtual Reality and Mental Health: Opportunities to Advance Research and Practice

\section{OPEN ACCESS}

Edited and reviewed by: Albert Rizzo,

University of Southern California, United States

${ }^{*}$ Correspondence: Nilufar Baghaei

N.Baghaei@massey.ac.nz

Specialty section:

This article was submitted to Virtual Reality in Medicine,

a section of the journal

Frontiers in Virtual Reality

Received: 17 December 2021

Accepted: 03 January 2022

Published: 03 February 2022

Citation:

Baghaei N, Liang H-N, Billinghurst M, Naslund $\mathrm{J}$ and Oyekoya O (2022) Editorial: Virtual Reality and Mental Health: Opportunities to Advance Research and Practice. Front. Virtual Real. 3:838036 doi: 10.3389/frvir.2022.838036
The World Health Organization predicts that by the year 2030, mental health conditions will be the leading burden of disease globally (World Health Organization, 2021). Mental health services around the world are struggling to meet the needs of users and fail to reach the majority of those in need. Preventative and early intervention, support and education can have significant positive impact on a person's prognosis (Health.vic, 2021). Technology offers new opportunities for reaching individuals who might not otherwise seek help due to difficulties in physically visiting health care professionals or fear of being stigmatised (Baghaei et al., 2020).

There has recently been a growing interest in using Virtual Reality (VR) technologies for enhancing people's mental health (Baghaei et al., 2021a; Xu et al., 2021). Advances in VR have created opportunities for collaboration between technology and health researchers, and practitioners to design, develop and evaluate tools to train and support healthcare providers, connect users with healthcare providers, provide access to affordable self-assessment, and to provide treatment or preventative strategies (Baghaei et al., 2021b). In this special issue, "Virtual Reality and Mental Health: Opportunities to Advance Research and Practice" (https://www.frontiersin.org/researchtopics/14974/virtual-reality-and-mental-health-opportunities-to-advance-research-and-practice), we bring together a collection of seven interesting research papers from diverse regions, including Germany (Rockstroh et al.; Döllinger et al.; Unruh et al.), the United Kingdom (Dilgul et al.), Ireland (Carroll et al.), the United States (Oyekoya et al.) and Australia (Kelly et al.) that are focused on the role of emerging VR technologies for advancing mental health research.

In the first study, Rockstroh et al. report on a room-scale VR experience for restorative walking, which they then evaluated in a randomised controlled experiment. The treatment condition with a room-scale approach resulted in increased user involvement, higher present moment awareness, increased perceived restorativeness of the experience, better subjective restoration, and an improved positive affect. The results suggest that room-scale VR in conjunction with virtual restorative environments could create sophisticated virtual restorative walks for confined spaces.

The second study focused on Virtual Reality Group Therapy (VRGT) for the treatment of depression. Dilgul et al. conducted semi-structured interviews to explore stakeholder views on VRGT. The patients used an avatar to interact with each other and with their therapist via a multiuser VR system. Therapists and patients responded favourably to the idea of doing group therapy sessions in VR. They both indicated that the anonymity provided by avatars could increase patients' willingness to talk more freely and honestly, which could increase participation and better group cohesion. 
In the third study, Döllinger et al. presents the results of a systematic review that explored the challenges and opportunities of immersive technologies for mindfulness meditation. The results showed that empirical research on XR-based mindfulness support mainly focuses on therapy and therapeutic outcomes. An analysis of empirical research on those systems did not reveal differences in mindfulness compared to non-mediated mindfulness practices. Furthermore, most of the currently investigated XR-supported mindfulness interactions are limited to vocally guided meditations within nature-inspired virtual environments, and so there are opportunites for more research in this area.

The fourth study focused on conducting a scoping review of AR/VR health and wellbeing interventions for older adults' physical and mental health. Carroll et al. argue that over $70 \%$ of the studies included in the review were mislabelled as VR, and only six papers included fully immersive VR/AR. The remaining studies use less immersive variants of VR with their populations, and only one study made use of AR, which prompted the suggestion of a new definition for Virtual Reality. This paper also calls for an updated taxonomy of AR and VR definitions to address the lack of consistency found in studies that identify themselves as AR/VR when they are using less immersive technological set-ups.

In the fifth study, Unruh et al. present an experiment investigating the difference in time experience with and without a virtual body in VR. They conducted a betweensubjects design with and without avatar embodiment as well as a real condition (i.e., avatar vs no-avatar vs real). A group of healthy subjects had to wait for seven and a half minutes in a room without any distractors or time indicators (e.g., clocks, sunlight). Participants were unaware that they would be later asked to estimate their waiting time duration and to describe their experience of the passage of time. They found that the presence of an avatar led to a significantly faster perceived passage of time. It seems to be promising to integrate avatar embodiment in future VR time-based therapy applications as they potentially could modulate a user's perception of the passage of time.

The sixth study explored first-person perspectives in designing a role-playing VR simulation for bullying prevention. Oyekoya et al. developed a simulation that allowed students to view themselves in different roles played in bullying situations in a VR setting. The expectation was that through role-playing, the students would explore different perspectives and learn how to respond to bullying situations. Findings from the focus group

\section{REFERENCES}

Baghaei, N., Chitale, V., Hlasnik, A., Stemmet, L., Liang, H.-N., and Porter, R. (2021). Virtual Reality for Supporting the Treatment of Depression and Anxiety: Scoping Review. JMIR Ment. Health 8 (9), e29681. doi:10.2196/29681

Baghaei, N., Stemmet, L., Hlasnik, A., Emanov, K., Hach, S., Naslund, J. A., et al. (2020). “Time to Get Personal: Individualised Virtual Reality for Mental studies of anti-bullying experts suggest that to create effective bullying prevention, VR simulations should consider focusing on role-playing, customization of the characters, environments, scenarios, and a scoring/reward system.

The final study in this special topic, from Kelly et al., described the iterative design and evaluation of Place, a VR app that supports mindfulness practice by situating the user in a virtual forest environment. Mindfulness practice was defined as bringing one's attention to the present moment and noticing events as they unfold with a non-judgmental attitude of acceptance. Their findings from focus groups drew attention to factors that influenced the user experience and acceptance of VR for mindfulness, and the authors described how the design was altered to address these factors.

These studies demonstrate that VR technologies not only appear acceptable and feasible for improving people's mental health but may also be a preferred medium for accessing support among many, due to the anonymity that it provides and extra features that are sometimes hard to replicate in real life. It is predicted that VR technology will be more popular and widely used in the future due to the significant drop in the cost and the increase in the quality of VR headsets. These studies emphasise the importance of carefully conducted pilot, focus group and exploratory studies to establish the feasibility and acceptability of the technology, and in particular, the value of involving potential users throughout the design, development and testing of VR interventions for supporting their mental health.

We have identified some gaps in the literature. While there have been extensive studies on VR therapy for pain management, anxiety, and phobia, there has been little work on supporting and treating depression using VR. There has also been little work on diagnosing mental health conditions using the technology (as opposed to providing therapy). Continued efforts are also necessary to strive toward inclusiveness in the design and development of VR interventions. Such efforts have the potential to promote adoption across diverse contexts, regions, and cultures, and ensure the generalisability of VR therapy toward reducing the burden of mental disorders globally.

\section{AUTHOR CONTRIBUTIONS}

All authors listed have made a substantial, direct, and intellectual contribution to the work and approved it for publication.

Health," in 2020 CHI Conference on Human Factors in Computing Systems (CHI EA '20), Hawaii, USA, April 25-30. (New York, NY, USA: Association for Computing Machinery), 1-9. doi:10.1145/3334480.3382932

Baghaei, N., Stemmet, L., Khaliq, I., Ahmadi, A., Halim, I., Liang, H. N., et al. (2021). "Designing Individualised Virtual Reality Applications for Supporting Depression: A Feasibility Study," in ACM SIGCHI Symposium on Engineering Interactive Computing Systems (EICS 2021), Eindhoven, Netherlands, June 8-11, 6-11. doi:10.1145/3459926.3464761 
Health.vic (2021). Early Intervention in Mental Illness. Available online at: https:// www2.health.vic.gov.au/mentalhealth/prevention-and-promotion/earlyinterventionin-mental-health (accessed December, 2021).

World Health Organization (2021). Mental Health. Available online at: http:// www.who.int/mental_health/en/(accessed December, 2021).

Xu, W., Liang, H.-N., Baghaei, N., Ma, X., Yu, K., Meng, X., et al. (2021). Effects of an Immersive Virtual Reality Exergame on University Students' Anxiety, Depression, and Perceived Stress: Pilot Feasibility and Usability Study. JMIR Serious Games 9 (4), e29330. doi:10.2196/29330

Conflict of Interest: The authors declare that the research was conducted in the absence of any commercial or financial relationships that could be construed as a potential conflict of interest.
Publisher's Note: All claims expressed in this article are solely those of the authors and do not necessarily represent those of their affiliated organizations, or those of the publisher, the editors and the reviewers. Any product that may be evaluated in this article, or claim that may be made by its manufacturer, is not guaranteed or endorsed by the publisher.

Copyright (๑) 2022 Baghaei, Liang, Billinghurst, Naslund and Oyekoya. This is an open-access article distributed under the terms of the Creative Commons Attribution License (CC BY). The use, distribution or reproduction in other forums is permitted, provided the original author(s) and the copyright owner(s) are credited and that the original publication in this journal is cited, in accordance with accepted academic practice. No use, distribution or reproduction is permitted which does not comply with these terms. 